\title{
Modified spherical harmonics method for one-speed transport equation with anisotropic scattering
}

\author{
M. S. Li \& B. Yang \\ Institute of Applied Physics and Computational Mathematics, Beijing, \\ People's Republic of China
}

\begin{abstract}
The criticality type eigenvalues of the one-speed transport equation in a homogeneous slab with anisotropic scattering and Marshak boundary conditions have been studied. The scattering function is assumed to be a combination of linearly anisotropic and strongly forward-backward scattering. When the forward and backward scattering completely dominate over the 'ordinary' scattering, or the thickness of the slab approaches zero, the highly peaked angular flux at the central point of the slab was expressed by finite width delta functions. Using the finite width delta functions to analyse the high-order truncation error of the angular flux we could accurately obtain results with a low-order approximation. Numerical results for critical eigenvalues are obtained and tabulated for different scattering parameters including the extreme cases, while the standard spherical harmonics method gets a singularity.
\end{abstract}

Keywords: spherical harmonics method, anisotropic scattering, finite width delta functions.

\section{Introduction}

Criticality type eigenvalues are needed for a variety of applications in reactor physics. The problem of anisotropy and its effects on the size of the system is one of the most important problems of transport theory. Many methods for computing transport equations have been proposed, such as the spherical harmonics $\left(P_{N}\right)$ method $[1,2]$ and the discrete ordinates $\left(S_{N}\right)$ method $[3,4,5]$. When the forward and backward scattering completely dominate over the 'ordinary' scattering (the extreme case) or the thickness of the slab approaches to 
zero, the angular distribution is very strongly peaked along the direction parallel to the slab surface, one has to take high-order functions into account for the highly peaked angular flux. The standard $P_{N}$ method is thus inadequate for this type of problem $[1,6]$. D. C. Sahni has solved the problem by combining the $S_{N}$ method, integral equation method and eigenvalue method [6]. To the best knowledge of the present author there is currently no article on completely calculating various combinations of the scattering parameters including the extreme case.

This work is to propose an easy-to-use comprehensive modified spherical harmonics method for computing transport equations in this situation. We have derived the asymptotic solution of transport equations and expressed the angular flux at the central point of the slab by finite width delta functions. Using the finite width delta functions to analyse the high-order truncation error of the angular flux we could accurately obtain results with low-order approximation. In this article we introduce our opinion in physics at first, then discuss the computational method, at last give results on several typical models and compare our results with the results calculated by the integral equation method.

\section{Transport equation}

With conventional notation [6], the starting linear transport equation for neutrons of one speed can be written as

$$
\Omega \cdot \nabla \psi(r, \Omega)+\Sigma_{t} \psi(r, \Omega)=c \Sigma_{t} \int \psi(r, \Omega) f\left(\Omega^{\prime} \cdot \Omega\right) d \Omega^{\prime} .
$$

The scattering kernel is assumed to be of the form

$$
f\left(\Omega^{\prime} \cdot \Omega\right)=\frac{1-\alpha-\beta}{4 \pi}\left(1+3 b_{1} \Omega^{\prime} \cdot \Omega\right)+\frac{\alpha}{2 \pi} \delta\left(\Omega^{\prime} \cdot \Omega-1\right)+\frac{\beta}{2 \pi} \delta\left(\Omega^{\prime} \cdot \Omega+1\right)
$$

here $0 \leq \alpha, \beta \leq 1, \alpha+\beta \leq 1$, and $\left|b_{1}\right| \leq 1 / 3$. In the present investigation we apply the theory to a source free, symmetric homogeneous infinite slab of thickness $2 a$. When this scattering function is inserted into eqn (1) we obtain the transport equation

$$
\begin{aligned}
\mu \frac{\partial \psi(x, \mu)}{\partial x} & +(1-\alpha c) \psi(x, \mu) \\
& =\frac{c}{2}(1-\alpha-\beta) \int_{-1}^{1}\left(1+3 b_{1} \mu \mu^{\prime}\right) \psi\left(x, \mu^{\prime}\right) d \mu^{\prime}+\beta c \psi(x,-\mu) .
\end{aligned}
$$

Here $x$ is the spatial variable measure in mean-free path, $\mu$ is the direction cosine of the angle between the positive $x$ axis and the neutrons velocity vector $\Omega$. The boundary conditions are that no neutrons enter the slab from the outside, i.e.

$$
\begin{cases}\psi(a, \mu)=0 & \mu<0 \\ \psi(-a, \mu)=0 & \mu>0\end{cases}
$$

When $\alpha, \beta, b_{1}$ and $c$ are specified we can solve critical thickness $2 a$. 


\section{Asymptotic analytic method and integral method}

Following Inonu [7], we divide the ordinary part of the scattering function into one symmetric and one antisymmetric part. We make the substitutions [6]

$$
\kappa(x, \mu)=\psi(x, \mu)-\frac{1-\alpha c-\gamma}{\beta c} \psi(x,-\mu)
$$

where

$$
\begin{gathered}
\tilde{c}=\frac{c(1-\alpha-\beta)}{1-c(\alpha+\beta)} \\
\gamma=\sqrt{(1-\alpha c)^{2}-\beta^{2} c^{2}} .
\end{gathered}
$$

Obviously, for $\gamma$ to be real it is required that $(\alpha+\beta) c \leq 1$.

With these substitutions we find that $\kappa(x, \mu)$ is a solution of the equation

$$
\mu \frac{\partial \kappa(x, \mu)}{\partial x}+\kappa(x, \mu)=\frac{\tilde{c} \gamma}{2} \int_{-1}^{1}\left(1+3 \tilde{b}_{1} \mu \mu^{\prime}\right) \kappa\left(x, \mu^{\prime}\right) d \mu^{\prime}
$$

where

$$
\tilde{b}_{1}=\frac{1-\alpha c-\beta c}{1-\alpha c+\beta c} b_{1} .
$$

The boundary conditions for the transformed flux $\kappa(x, \mu)$ will then be

$$
\begin{cases}\kappa(a, \mu)=R \kappa(a,-\mu) & \mu<0 \\ \kappa(-a, \mu)=R \kappa(-a,-\mu) & \mu>0 .\end{cases}
$$

where

$$
R=\frac{\beta c}{1-\alpha c+\gamma} .
$$

Eliminating $\psi(\tau,-\mu)$ from eqn (5) with arguments $\mu$ and $-\mu$ we obtain

$$
\begin{gathered}
\psi(x, \mu)=\frac{1-\alpha c+\gamma}{2 \gamma} \kappa(x, \mu)+\frac{\beta c}{2 \gamma} \kappa(x,-\mu) . \\
\int_{-1}^{1} \psi(x, \mu) d \mu=\frac{1-\alpha c+\beta c+\gamma}{2 \gamma} \int_{-1}^{1} \kappa(x, \mu) d \mu .
\end{gathered}
$$

Utilizing the symmetry of the angular flux at $x=0 \quad \psi(0, \mu)=\psi(0,-\mu)$ we obtain

$$
\psi(0, \mu)=\frac{1-\alpha c+\beta c+\gamma}{2 \gamma} \kappa(0, \mu) .
$$

From eqns (13) and (14) we know the zero moments of the angular flux $\psi_{0}(x)=\int_{-1}^{1} \psi(x, \mu) d \mu, \kappa_{0}(x)=\int_{-1}^{1} \kappa(x, \mu) d \mu$ as a function of $x$ have a similar distribution and $\psi(0, \mu), \kappa(0, \mu)$ as a function of $\mu$ have a similar distribution.

If we assume that $b_{1}=0$ and

$$
\kappa_{0}(x)=\cos (x / \eta) .
$$


When eqn (15) is inserted into eqn (8) we obtain

$$
k(0, \mu)=\frac{\tilde{c}}{2} \frac{\left(e^{\frac{2 a \gamma}{|\mu|}}-R\right)+\left[(R-1) \cos \left(\frac{a}{\eta}\right)+(R+1)\left(\frac{|\mu|}{\gamma \eta}\right) \sin \left(\frac{a}{\eta}\right)\right] e^{\frac{a \gamma}{|\mu|}}}{\left[1+\left(\frac{\mu}{\gamma \eta}\right)^{2}\right]\left[e^{\frac{2 a \gamma}{|\mu|}}-R\right]} .
$$

(a) For $\beta=0$ from eqn (11) it follows that $R=0$

$$
k(0, \mu)=\frac{\tilde{c}}{2} \frac{1-\cos (a / \eta) e^{-a \gamma /|\mu|}+\sin (a / \eta) e^{-a \gamma /|\mu|}}{1+(\mu / \gamma \eta)^{2}}
$$

When $\alpha \rightarrow 1 / c$ and $a / \eta \rightarrow 0$ if we assume the scale flux as a function of $x$ is a constant we obtain

$$
\begin{gathered}
k(0, \mu)=\frac{\tilde{c}}{2}\left(1-e^{-a \gamma /|\mu|}\right) . \\
\kappa_{0}(0)=\tilde{c} \gamma\left[1-e^{-a \gamma}+a \gamma E_{1}(a \gamma)\right]
\end{gathered}
$$

where $E_{1}(x)$ is exponential integral function. Neglecting terms proportional to $\gamma$ and of higher order

$$
\begin{gathered}
\tilde{c} \gamma a\left[1-\gamma_{0}-\log (\gamma a)\right] \simeq 1 \\
c a(1-\alpha)\left\{1-\gamma_{0}-\log [(1-\alpha c) a]\right\} \simeq 1
\end{gathered}
$$

where $\gamma_{0}$ is Euler's constant $\gamma_{0}=0.577216$.

(b) For $\beta \neq 0$ when $\alpha+\beta \rightarrow 1 / c$ from eqn (11) it follows $R=-1, \kappa_{0}(a)=0$, we obtain $\cos (a / \eta) \approx 0$ and $\sin (a / \eta) \approx 1$

$$
\begin{aligned}
& k(0, \mu)=\frac{\tilde{c}}{2} \frac{1}{1+(\mu / \gamma \eta)^{2}} . \\
& k_{0}(0)=\tilde{c} \gamma \eta \operatorname{arctg}(1 / \gamma \eta) .
\end{aligned}
$$

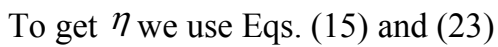

$$
\tilde{c} \gamma \eta \operatorname{arctg}(1 / \gamma \eta)=1 \text {. }
$$

This is close to the discrete eigenvalues of the Case spectrum $\left[{ }^{8}\right]$.

It shows that the expression $2 c a(1-\alpha-\beta)(1-\alpha c+\beta c) / \gamma$ approaches a constant when $\alpha+\beta \rightarrow 1 / c$. The limiting value is 2 (1.954 in reference [6]).

$$
2 c a(1-\alpha-\beta)(1-\alpha c+\beta c) / \gamma=2.0 \text {. }
$$

(c) As shown by Siewert and Williams [9] and Sahni et al [6] eqn (8) can be transformed into an integral equation for the zero moments of the angular flux $\kappa_{0}(x)$. If we assume that $b_{1}=0$ and $\kappa_{0}(x)$ is an even function of $x$ it will read 


$$
\begin{aligned}
\kappa_{0}(x)= & \frac{\tilde{c} \gamma}{2} \int_{-a}^{a} \kappa_{0}(y) E_{1}(\gamma|x-y|) d y+\frac{\tilde{c} \gamma}{2} \int_{-a}^{a} \kappa_{0}(y) d y \\
& \times \int_{0}^{1} \frac{\exp \left(\frac{-2 a \gamma}{\mu}\right)\left\{\exp \left[\frac{(y-x) \gamma}{\mu}\right]+\exp \left[\frac{(y+x) \gamma}{\mu}\right]\right\} d \mu}{\mu[1-R \exp (-2 a \gamma / \mu)]} .
\end{aligned}
$$

For $\beta=0$ with a constant scale flux assumption eqn (26) will be

$$
\kappa_{0}(0)=\frac{\tilde{c} \gamma}{2} \int_{-a}^{a} E_{1}(\gamma|y|) d y .
$$

Expand $E_{1}(x)$ at $x=0$ and neglecting terms proportional to $\gamma$ and of higher order, we could obtain eqs. (20) and (21).

\section{Spherical harmonics method}

For the Spherical harmonics method solution, the angular flux is expanded in a series of Legendre polynomials as

$$
\psi(x, \mu)=\sum_{n=0}^{N}\left(\frac{2 n+1}{2}\right) \Phi_{n}(x) P_{n}(\mu) .
$$

This expansion (28) can now be substituted into eqn (3) in order to obtain the function $\Phi_{n}(x)$. Multiplying both sides of the resulting equation by $P_{m}(\mu)$, integrating overt and utilizing the orthogonally properties and the recursion relations of the Legendre polynomials, after some rearrangement we have:

$$
\begin{aligned}
n & \frac{d \Phi_{n-1}(x)}{d x}+(n+1) \frac{d \Phi_{n+1}(x)}{d x}+(2 n+1) \Phi_{n}(x) \\
= & (2 n+1)\left[c(1-\alpha-\beta) \Phi_{0}(x) \delta_{n 0} b_{1} c(1-\alpha-\beta) \Phi_{1}(x) \delta_{n 1}\right. \\
& \left.+\alpha c \Phi_{n}(x)+\beta c(-1)^{n} \Phi_{n}(x)\right] \quad n=0,1,2, \ldots N .
\end{aligned}
$$

One may employ the well-known procedure of seeking a solution of the homogeneous eqn (29) in the form

$$
\Phi_{n}(x)=G_{n}(v) \exp (-x / v)
$$

where the $G_{n}(v)$ are some constants. Each of the $\Phi_{n}(x)$ defined in eqn (30) will satisfy eqn (29) provided that the characteristic $P_{N}$ equations are satisfied:

$$
\begin{gathered}
(n+1) G_{n+1}(v)+n G_{n-1}(v)-(2 n+1) v\{1-c(1-\alpha-\beta) \\
\left.\times\left(\delta_{n 0}+b_{1} \delta_{n 1}\right)-c\left[\alpha+(-1)^{n} \beta\right]\right\} G_{n}(v)=0 .
\end{gathered}
$$

Eqn (31) has a homogeneous matrix form:

$$
[\mathbf{M}(v)] \mathbf{G}=0 .
$$


The coefficient matrix is $(N+1) \times(N+1)$, and $\mathbf{G}=\left[G_{0}, G_{1} \ldots G_{n}\right]^{T}$. The unknown constant vector $\mathrm{G}$ is determined from the normalization $G_{-1}(v)=0$ and $G_{0}(v)=1$. The general solutions of eqn (3) with $N$ odd can be expressed, for $n=0,1, \cdots N$, as

$$
\Phi_{n}(x)=\sum_{j=1}^{(N+1) / 2} G_{n}\left(v_{j}\right)\left[A_{j} F_{n}\left(-x / v_{j}\right)+(-1)^{n} B_{j} F_{n}\left(x / v_{j}\right)\right]
$$

where $G_{n}(-v)=(-1)^{n} G_{n}(v), F_{n}\left( \pm x / v_{j}\right)=\exp \left( \pm x / v_{j}\right)$ and $A_{j}$ and $B_{j}$ are

coefficients to be determined from the boundary and symmetry conditions of the problem. The essential idea of the standard $P_{N}$ method is that $\Phi_{N+1}(v)=0$, i.e. the permissible eigenvalue $v_{j}$ is the jth positive zero of $G_{N+1}(v)$. The determination of the roots is obtained using the Newton-Raphson iterative technique.

For a finite slab of half-thickness $a$ we apply the Marshak boundary condition

$$
\int_{0}^{1} P_{2 k-1}(\mu) \psi(a,-\mu) d \mu=0 \quad k=1,2, \ldots(N+1) / 2
$$

to the general solution (31), and then obtain an eigenvalue equation which relates the critical dimensions $a$ to the parameter $c$, or vice versa. This can be written in matrix form as

$$
[\mathbf{M}(a)] \mathbf{A}=[0]
$$

where $\mathbf{A}$ is a vector with elements $\mathbf{A}_{k}, k=1,2, \ldots(N+1) / 2$ and the $[(N+1) / 2]^{2}$ elements of matrix $\mathbf{M}_{k, j}(a)$ are given by

$$
\mathbf{M}_{k, j}(a)=\sum_{n=0}^{(N-1) / 2} \varphi(n, k) G_{2 n}\left(v_{j}\right) \cosh \left(a / v_{j}\right)+G_{2 k-1}\left(v_{j}\right) \sinh \left(a / v_{j}\right)
$$

where

$$
\varphi(n, k)=\frac{(4 n+1)(-1)^{n+k}(2 k-1) !(2 n) !}{2^{2 k+2 n-1}(2 n-2 k+1)(k+n)[n !(k-1) !]^{2}} .
$$

Clearly eqn (37) is a linear system of algebraic equations. This system has a non-trivial solution (for $\mathbf{A}_{k}$ ) if determinant $\mathbf{M}_{k, j}(a)$ vanishes, and this condition yields the desired results.

\section{Modified spherical harmonics method}

The $P_{N}$ approximation ( $N$ odd) consists of truncating the expansion (28) after $N+1$ terms, i.e. at $n=N$. One also chooses a finite number of points of the $v$ spectrum, given by the roots of the eqn (31) and $G_{N+1}(v)=0$. Since $c>1$, the root $v_{1}$ is imaginary (we let $v_{1}=i \eta$ ) and very close to the discrete eigenvalue $v_{0}$ of the Case spectrum [8]. The eigenfunction corresponding imaginary 
eigenvalue $v_{1}$ is dominant part of the angular flux when $\alpha+\beta$ approaches the value $1 / c$.

It is necessary to count for the high-order terms of eqn (28) as the angular flux is a very strongly peaked function along the direction parallel to the slab surface. The modified spherical harmonics $\left(\delta P_{N}\right)$ method is that using the finite width delta functions analyse the high-order truncation error of the angular flux and the high-order part of the angular flux is calculated analytically.

$$
G_{n}\left(v_{1}\right)=D_{n} \quad \text { for } n \in \text { even and } n \geq N+1
$$

where

$$
D_{n}=\int_{-1}^{1} \delta(\mu) P_{n}(\mu) d \mu .
$$

From the previous section we know the finite width delta functions $\delta(\mu)$ are expressed by eqns (18) and (22) for $\beta=0$ and $\beta \neq 0$ separately.

$$
\delta(\mu)= \begin{cases}\frac{1}{2} \frac{1-e^{-a \gamma /|\mu|}}{1-e^{-a \gamma}+a \gamma E_{1}(a \gamma)} & \text { if } \beta=0 ; \\ \frac{1}{2 \gamma \eta \operatorname{arctg}(1 / \gamma \eta)} \frac{1}{1+(\mu / \gamma \eta)^{2}} & \text { if } \beta \neq 0 .\end{cases}
$$

The value of $G_{N+1}\left(v_{1}\right)$ is not equal to zero at this time

$$
G_{N+1}\left(v_{1}\right)=D_{N+1} .
$$

To apply the Marshak boundary condition (34) we must take into account the high-order parts of angular flux so eqn (36) for $j=1$ will be changed to

$$
\begin{aligned}
M_{k, 1}(a)= & \left(\sum_{n=0}^{(N-1) / 2} \varphi(n, k) G_{2 n}\left(v_{1}\right)+\sum_{n=(N+1) / 2}^{\infty} \varphi(n, k) D_{2 n}(\eta)\right) \\
& \times \cos (a / \eta)+G_{2 k-1}\left(v_{1}\right) \sin (a / \eta) . \\
M_{k, 1}(a)= & \left(\sum_{n=0}^{(N-1) / 2} \varphi(n, k)\left(G_{2 n}\left(v_{1}\right)-D_{2 n}(\eta)\right)+\int_{0}^{1} P_{2 k-1}(\mu) \delta(\mu) d \mu\right) \\
& \times \cos (a / \eta)+G_{2 k-1}\left(v_{1}\right) \sin (a / \eta) .
\end{aligned}
$$

\section{Numerical results and discussion}

In order to examine the validity and accuracy of the present method two computer programs ( $\delta P_{N}$ and integrate method) in FORTRAN were written to calculate the critical thickness. We took the results from integral equation method as reference values.

The integral eqn (26) was solved iteratively by substituting improved flux approximation in to the right-hand side of the equation. When $\alpha+\beta \rightarrow 1 / c$ the angular flux $\kappa(x, \mu)$ are high-order functions of $\mu$ for $\beta \neq 0$ we use $N=24$ to 520 double-Gauss quadrature sets for different combinations $\alpha, \beta$ and $c$. 
Table 1: The critical slab thickness $2 a$ obtained in $P_{N}, \delta P_{N}$ approximation and integrate (Int ) method with different value of $c, \alpha, \beta$ and degree of linearly anisotropic scattering comparison with results of C. Yildiz [1].

\begin{tabular}{|c|c|c|c|c|c|c|}
\hline$c, \alpha, \beta$ & $b_{1}$ & $P_{11}$ & $\delta P_{3}$ & $\delta P_{11}$ & Int & $P_{11} P_{13}^{*}[1]$ \\
\hline \multirow{3}{*}{$\begin{array}{c}1.2 \\
0.70,0.0\end{array}$} & -0.3 & 2.95114 & 2.95434 & 2.93843 & \multirow{3}{*}{3.08555} & 2.95115 \\
\hline & 0 & 3.11594 & 3.14486 & 3.10476 & & 3.11595 \\
\hline & 0.3 & 3.32199 & 3.34232 & 3.31169 & & 3.32199 \\
\hline \multirow{3}{*}{$\begin{array}{c}1.2 \\
0.8333,0.0\end{array}$} & -0.3 & 2.60780 & 0.86736 & 0.87132 & \multirow{3}{*}{0.89557} & 2.60780 \\
\hline & 0 & 2.66236 & 0.87926 & 0.87928 & & 2.66236 \\
\hline & 0.3 & $-{ }_{-1}$ & -- & - & & 2.72127 \\
\hline \multirow{3}{*}{$\begin{array}{c}2.0 \\
0.49,0.0\end{array}$} & -0.3 & 0.52586 & 0.31261 & 0.31780 & \multirow{3}{*}{0.32932} & 0.52586 \\
\hline & 0.0 & 0.53742 & 0.32049 & 0.32095 & & 0.53742 \\
\hline & 0.3 & -- & -- & -- & & 0.54996 \\
\hline \multirow{3}{*}{$\begin{array}{c}2.0 \\
0.4999,0.0\end{array}$} & -0.3 & 0.52156 & 0.17348 & 0.17428 & \multirow{3}{*}{0.17913} & 0.52156 \\
\hline & 0.0 & 0.53247 & 0.17586 & 0.17587 & & 0.53247 \\
\hline & 0.3 & -- & -- & -- & & 0.54425 \\
\hline \multirow{3}{*}{$\begin{array}{c}1.2 \\
0.0,0.80\end{array}$} & -0.3 & 1.14702 & 0.97472 & 1.02865 & \multirow{3}{*}{1.01267} & 1.14702 \\
\hline & 0 & 1.15204 & 0.98166 & 1.03315 & & 1.15204 \\
\hline & 0.3 & 1.15715 & 0.98875 & 1.03772 & & 1.15715 \\
\hline \multirow{3}{*}{$\begin{array}{c}1.2 \\
0.0,0.8333\end{array}$} & -0.3 & 0.95199 & 0.00987 & 0.01707 & \multirow{3}{*}{0.04210} & 0.95199 \\
\hline & 0 & 0.95384 & 0.00987 & 0.01707 & & 0.95384 \\
\hline & 0.3 & 0.95569 & 0.00988 & 0.01708 & & 0.95569 \\
\hline \multirow{3}{*}{$\begin{array}{c}2.0 \\
0.0,0.49\end{array}$} & -0.3 & 0.33843 & 0.08039 & 0.11128 & \multirow{3}{*}{0.14402} & 0.33843 \\
\hline & 0.0 & 0.34093 & 0.08114 & 0.11188 & & 0.34093 \\
\hline & 0.3 & 0.34349 & 0.08191 & 0.11250 & & 0.34349 \\
\hline \multirow{3}{*}{$\begin{array}{c}2.0 \\
0.0,0.4999\end{array}$} & -0.3 & 0.33164 & 0.00204 & 0.00378 & \multirow{3}{*}{0.01852} & 0.33164 \\
\hline & 0.0 & 0.33389 & 0.00204 & 0.00378 & & 0.33389 \\
\hline & 0.3 & 0.33618 & 0.00204 & 0.00378 & & 0.33618 \\
\hline \multirow{3}{*}{$\begin{array}{c}1.5 \\
0.33,0.33\end{array}$} & -0.3 & 0.67499 & 0.16057 & 0.22206 & \multirow{3}{*}{0.28622} & $0.66691^{*}$ \\
\hline & 0 & 0.67993 & 0.16207 & 0.22326 & & $0.67168^{*}$ \\
\hline & 0.3 & 0.68501 & 0.16360 & 0.22449 & & 0.67658 \\
\hline \multirow{3}{*}{$\begin{array}{c}1.5 \\
0.33,0.3\end{array}$} & -0.3 & 0.73696 & 0.45872 & 0.55192 & \multirow{3}{*}{0.56570} & $0,73009^{*}$ \\
\hline & 0 & 0.74475 & 0.46617 & 0.55693 & & $0.73770^{*}$ \\
\hline & 0.3 & 0.75285 & 0.47388 & 0.56202 & & $0.74562 *$ \\
\hline \multirow{3}{*}{$\begin{array}{c}1.5 \\
0.42,0.15\end{array}$} & -0.3 & 0.93023 & 0.79248 & 0.88245 & \multirow{3}{*}{0.88135} & $0.92990^{*}$ \\
\hline & 0 & 0.95138 & 0.81434 & 0.90186 & & $0.95144 *$ \\
\hline & 0.3 & 0.97453 & 0.83624 & 0.92250 & & $0.97506^{*}$ \\
\hline
\end{tabular}

For the specified $\alpha, \beta, c$ and $b_{1}$ we first computed the eigenvalue $v_{1}=i \eta$ from eqn (24) and start the calculation with some assumed value of halfthickness $a$, then we could calculate $D_{n}\left(v_{1}\right)$ by eqn (39). Except $G_{N+1}\left(v_{1}\right)$ 
and $M_{k, 1}(a)$ the calculation procedures in present work are the same as standard $P_{N}$ method. After we obtain an improved value of half-thickness $a$ and continue this process until we get a convergence accurate result.

In table 1 , we present the critical slab thickness $2 a$ obtained in $P_{N}$ method, $\delta P_{N}$ method and integrate (Int) method with different value of $c, \alpha, \beta$ and degree of linearly anisotropic scattering $b_{1}$. In reference [1] Yildiz had compared the results calculated by standard $P_{N}$ method with the results of Sahni et al [6] and others. The conclusion was that the standard $P_{N}$ method could give reasonable results for thick slab. We directly compare our results obtained by standard $P_{N}$ method with those in reference [1]. The agreement is generally within five or six significant decimal places for $N<13$. For $N=13,15$ the agreement is generally within three or four significant decimal places. We couldn't obtain converged results for some combination of $\alpha, \beta$ when $\alpha+\beta \rightarrow 1 / c$ with $b_{1}=0.3$.

When $\gamma \approx 1$ the value of $G_{N+1}$ is nearly zero. The low-order $P_{N}$ approximations generally give accurate results for thick slabs [1,2]. For example when $\alpha=0.7$, $\beta=0.0, c=1.2, b_{1}=0.0, \gamma=0.16$ and $G_{12}=0.0066$ the $P_{N}, \delta P_{N}$ methods give closely results.

When the values $\alpha+\beta$ approach the limit value $1 / c$ the angular distribution is very strongly peaked along the plane of the slab. The critical slab thickness $2 a$ varies rapidly with $c$ and approaches to zero but the standard $P_{N}$ method give an unchanged result. For example while for $\alpha=0.0, \beta=0.4999, c=2.0, b_{1}=0.0$, $\gamma=0.02, G_{N+1}=0.2252$ the critical slab thickness $2 a$ are 0.33389 for $P_{11}$. The $\delta P_{N}$ method gives $2 a=0.00378$ for $\delta P_{11}$. Even $\delta P_{3}$ approximation could give a reasonable result $2 a=0.00204$. In table 1 we could see when $\alpha=0.8333$, $\beta=0.0, c=1.2, b_{1}=0.0, \gamma=4 \times 10^{-5}, G_{N+1}=0.164$ the critical slab thickness $2 a$ are $2.6623,0.87928$ for $P_{11}, \delta P_{11}$ respectively.

It is seen from Table 1 that the $\delta P_{N}$ method has more accuracy than standard $P_{N}$ method but has less accuracy than integral equation method. When $\alpha+\beta \rightarrow 1 / c$ the critical slab thickness $2 a$ as a function of $\alpha, \beta$ approaches to zero more rapidly than real. We think that the errors occur in the lack of taking account of the high-order moments of the angular flux in the boundary condition eqn (34).

\section{Conclusion}

In this paper we have considered the slab criticality problem of the linear transport equation with forward, backward and linearly anisotropic scattering in a homogeneous slab. Using the asymptotic analytic method we obtain the asymptotic angular flux which expressed by finite width delta functions at the central point of the slab when $\alpha+\beta$ approaches the value $1 / c$. Using the finite 
width delta functions to consider more terms in the expansion of angular flux we could obtain accurately results with low-order $N$ and less computational effort. The present scheme converges quite well and enables us to obtain accurate results even with the low-order formulas.

The present method is applicable to large system, and also to very small system. The slab thickness as a function of $\alpha, \beta$ are continuously and smoothly for various $\alpha+\beta$ ranging from 0 to the extreme case $1 / c$. The present method can also be extended to more general problems with two or three dimension high-order anisotropy and to multiregional energy dependent problems. We have started looking into these aspects and the conclusion awaits further work.

\section{Acknowledgement}

This work was partially supported by the National Key Laboratory of Computational Physics under Grant No.51479050105ZW0906.

\section{References}

[1] C. Yildiz, Variation of the critical slab thickness with the degree of strongly anisotropic scattering in one-speed neutron transport theory, Ann. Nucl. Energy, 1998; Vol. 25, No. 8, pp. 529 540,

[2] C. Yildiz. Influence of anisotropic scattering on the size of timedependent systems in monoenergetic neutron transport, Journal of Physics D 1999; 32: 317.

[3] R.C. Barros and E.W. Larsen. A numerical method for one group slab geometry discrete ordinates problems with no spatial truncation error. Nuclear Science and Engineering 1990; 104: 199.

[4] W. A. Fiveland. Three-dimensional radiative heat transfer solutions by the Discrete Ordinates Method. AIAA J. Thermophysics 1988; 2(4): 309-316.

[5] J. E. Morel, A hybrid collocation-Galerkin-Sn method for solving the Boltzmann transport equations. Nuclear Science and Engineering 1989; 101: $72-87$.

[6] D. C. Sahni, N. G. Sjostrand and N. S. Garis, Criticality and time eigenvalues for one-speed neutrons in a slab with forward and backward scattering. Journal of Physics D 1992; 25:1381.

[7] E. Inonu, Transport Theory and Statistical Physics 1973; 3: 137

[8] K. M. Case and P. F. Zweifel, Linear Transport Theory. Addison Wesley, Reading, MA, 1967.

[9] C. E. Siewert and M. M. R. Williams, The effect of anisotropic scattering on the critical slab problem in neutron transport theory using a synthetic kernel. Journal of Physics D 1977; 10: 2031-2040. 